\title{
MATHEMATICAL EXPLANATION TO SOLUTION FOR EX-NOR PROBLEM USING MLFFN
}

\author{
Vaibhav Kant Singh \\ Department of Computer Science and Engineering, Institute of Technology, Guru \\ Ghasidas Vishwavidyalaya, Central University, Bilaspur,Chhattisgarh,India
}

\begin{abstract}
This paper is in continuation to what we did previously in the field of Soft Computing. In the previous work the author proposed several solutions to the XOR problem. In this paper we will see some solutions to the Ex-NOR problem using the concept of MLFFN. Ex-NOR problem is a non-linearly separable problem the solutions of which are discussed in this paper. The Artificial Neural Network proposed is mathematically proved to be successful in providing solution to the Ex-NOR problem. The solutions proposed are explained by means of Architectural Graph and Signal flow graph.
\end{abstract}

\section{KEYWORDS}

Artificial Neural Network, Ex-NOR, Activation function, Signal Flow Graph \& Architectural Graph

\section{INTRODUCTION}

Soft Computing is the branch of Computer Science that deals with methodologies that solve the problems which portray imprecision, partial truth and uncertainty. In Soft Computing the impression is tolerable. Computational time when compared with Hard Computing is less due to the presence of intelligent computational steps. Soft Computing involves systems which are basically inspired from nature like Artificial Neural Network and Fuzzy Logic. The programs made are going to follow new laws which are going to justify the logic behind the programming done. The output produced need not to be deterministic in nature. It may allow parallel computation. It is capable to produce approximate result to the problem posed by the user. Soft Computing is basically a branch that deals with three known streams for the implementation of the idea that it portrays. The three streams are Artificial Neural Network, Fuzzy Systems and Genetic Algorithm and Evolutionary Programming.

In this paper the author Vaibhav Kant Singh focuses on the stream Artificial Neural Network. Artificial Neural Network (ANN) is a stream that deals with construction of systems which are having analogy in working with the Biological Neural Network working. The ANN systems are capable of mapping input to output. ANN systems are capable to get trained; they are capable to learn from previous examples. ANN is capable of generalization. ANN having resemblance with Biological Network of Neurons are robust and fault tolerant in nature. Some currently existing ANN systems are Adaptive Resonance Theory (ART), Bidirectional Associative Memory (BAM), Perceptron (Single Layer and Multilayer), ADALINE and MADALINE, Counter Propagation Network, Multi Layer Feed Forward Network (MLFFN), Hopfield Network, 
International Journal of Information Sciences and Techniques (IJIST) Vol.6, No.1/2, March 2016 Necognition, Self-Organizing feature map, Brain-State-In-A-Box, BPN, Boltzmann machines etc. Artificial Neural Network systems comprises of three basic elements namely the Individual neurons present in the ANN, the network topology (the network architecture) and the learning algorithm employed to train the ANN.

\section{LITERATURE SURVEY}

In [1] Vaibhav Kant Singh proposed one solution to the XOR problem using MLP. In [2] Vaibhav Kant Singh proposed two other solutions to the XOR problem. In [3] Vaibhav Kant Singh proposed solutions to the various logic gates. In [4] Vaibhav Kant Singh gave a survey to the various learning techniques employed for training the Artificial Neural Network. In $[5,6]$ Vaibhav Kant Singh proposed another solution to the XOR problem using minimum configuration MLP.

\section{Problem STATEMENT}

The Ex-NOR problem is non-linearly separable problem. The Definition of linear separability is two sets of points $A$ and $B$ in an $n$-dimensional space are called linearly separable if $(n+1)$ real numbers $w 1, w 2 \ldots w(n+1)$ exists, such that every point $(\mathrm{x} 1, \mathrm{x} 2 \ldots \ldots \mathrm{xn}) \in \mathrm{A}$ satisfies wixin $i=1 \geq w(n+1)$ exist and every point $(x 1, x 2 \ldots x n) \in B$ satisfies wixin $i=1<w(n+1)$ exist. Table 1 represents the Ex-NOR logic and the Figure in Table 1 specifies the portrayal of the inputs applicable for Ex-NOR problem in Two-dimensional plane. It is very clear from the table that it is not possible to divide the inputs into two classes on the basis of output. Thus from the research that the author Vaibhav Kant Singh [1,2,5] made earlier the problem is a non-linearly separable problem.

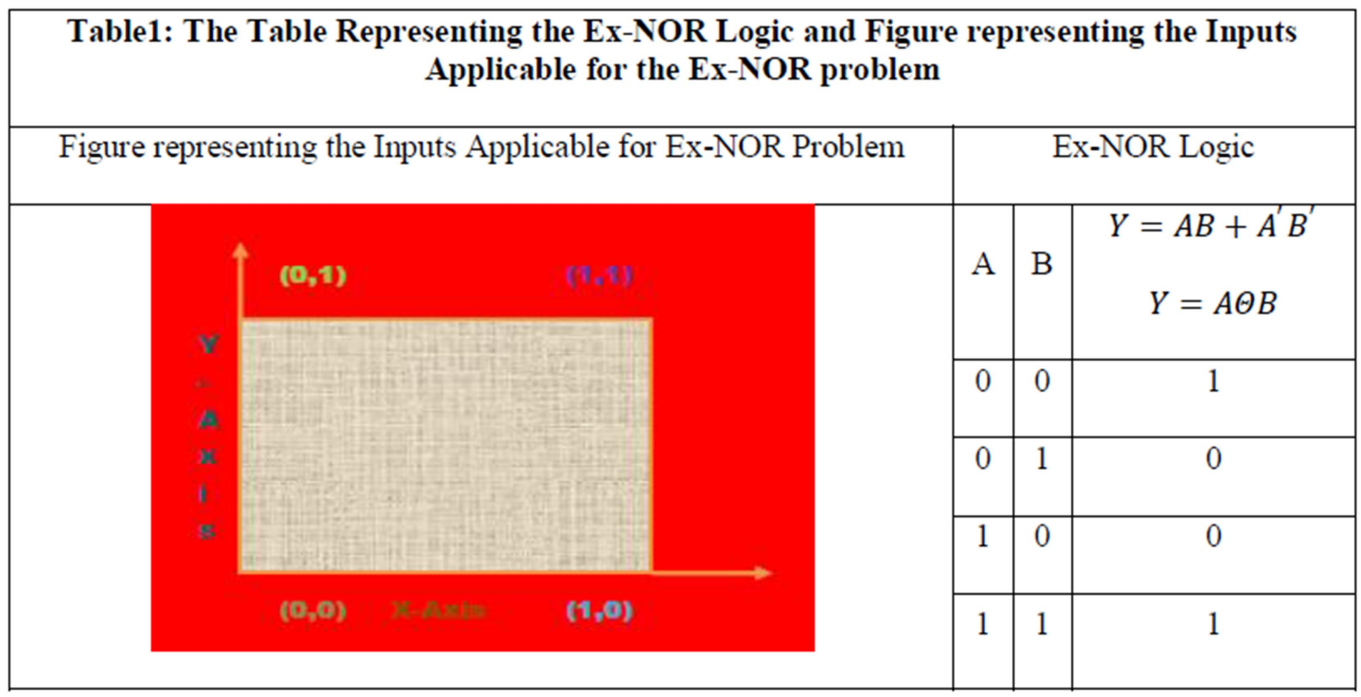




\section{Proposed SOLUTIONS}

In this paper we will see some solutions to the Ex-NOR problem using MLFFN.

\subsection{First Proposed Solution to the Ex-NOR problem}

In first proposed solution a MLFFN is proposed which comprises of three layers. The First layer comprises of two neurons. The second layer comprises of one neuron. The third layer comprises of one neuron. The first layer comprises of non-linear neuron. The second and third layer comprises of neurons which are having linear in nature. The neurons are characterized linear and non-linear on the basis of the characteristic they exhibit. The naming of the network could be (22-1-1). The solution proposed specifies the final values for weights acquired after training the network. After attaining the values prescribed in the solution the proposed ANN gives the desired values for output for the applicable input to the Ex-NOR logic GATE. In this section wewill see the mathematical proof of the provided solution. Figure 1 represents the proposed Architectural Graph for the problem, whereas the Figure 2 represents the Signal Flow Graph giving the detail of the weights and other parameters involved in the ANN. In the Architectural graph NL represents nonlinear whereas LE represents Linear element. Z represents the output produced by the ANN.

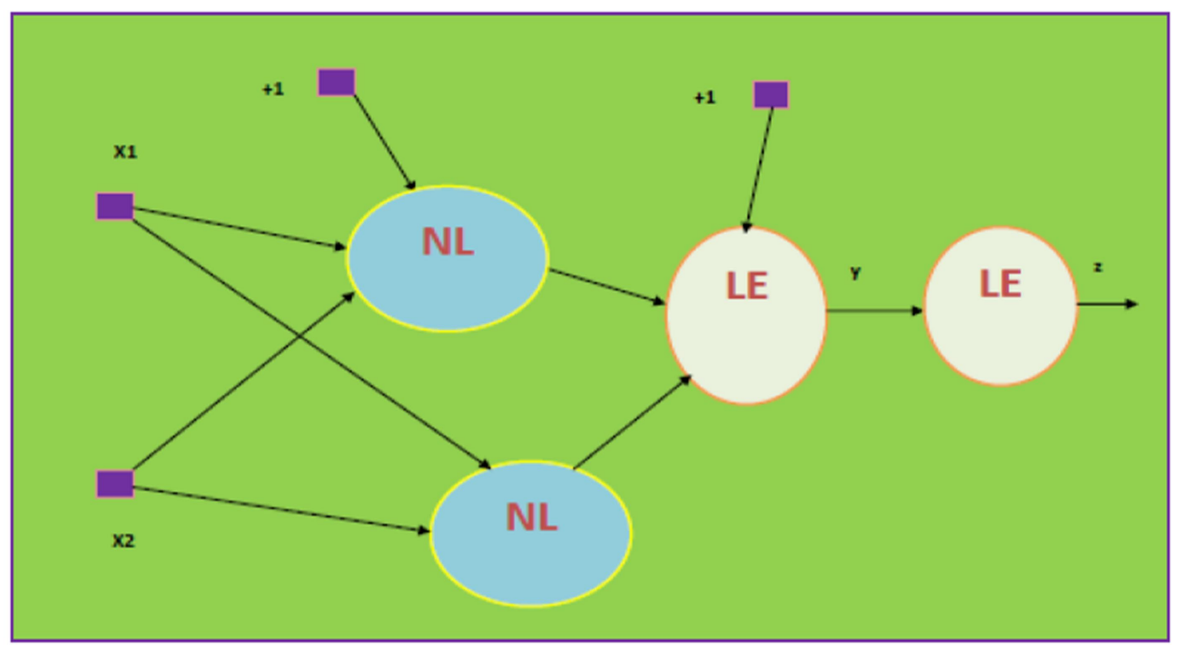

Figure1: Architectural Graph representing First and Third Solution to Ex-NOR Problem 


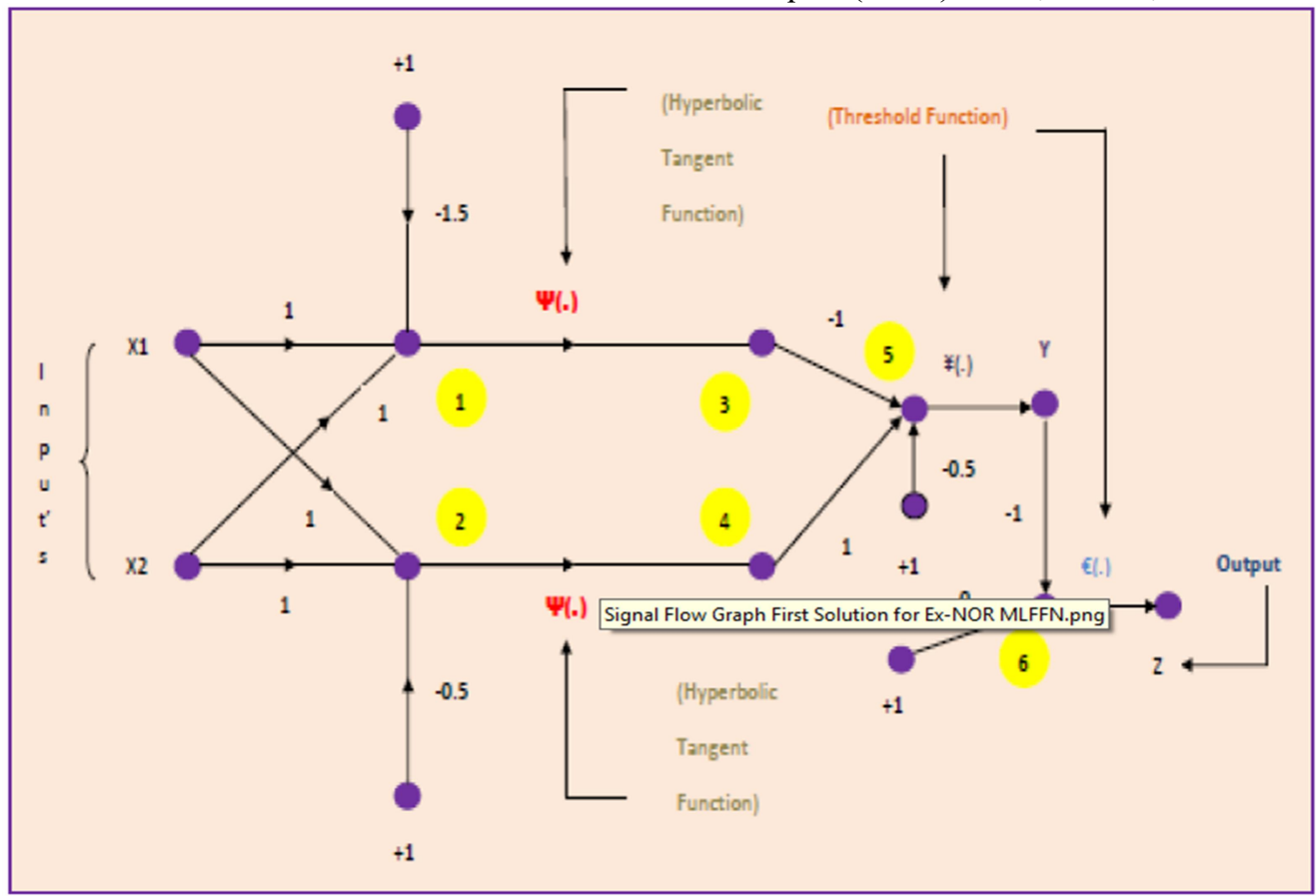

Figure2: Signal Flow Graph representing First Solution to Ex-NOR Problem

Here, the activation function used for non linear elements in the hidden layer is hyperbolic tangent function. Definition of hyperbolic tangent function is :-

$\tan h x=\frac{\sin h x}{\cos h x}=\frac{e^{x}-e^{-x}}{e^{x}+e^{-x}}=\frac{e^{2 x}-1}{e^{2 x}+1}=\frac{1-e^{-2 x}}{1+e^{-2 x}} \ldots$.

Here, $e=2.7182818456$ i.e. the natural logarithm base known as Euler's number, $x=$ induced local field value and Range of values for function will be $[-1,+1]$.At node 3 the signal value will be (From $\mathrm{Eq}(2) \& \mathrm{Eq}(5))$

$\Psi(-1.5)=\frac{1-e^{-2 \times(-1.5)}}{1+e^{-2 \times(-1.5)}}=\frac{-19.08553692}{21.08553692}=(-0.905148253)$

At node4 the signal value will be ( $\mathrm{From} \mathrm{Eq}(4) \& \mathrm{Eq}(5))$

$\Psi(-0.5)=\frac{1-e^{-2 \times(-0.5)}}{1+e^{-2 \times(-0.5)}}=\frac{-1.718281828}{3.718281828}=(-0.462117157)$

At node5 the signal value will be ( $\mathrm{From} \mathrm{Eq}(6) \& \mathrm{Eq}(7))$

node $5=$ node $3 \times-1+$ node $4 \times 1+1 \times(-0.5)$

node $5=(-0.9051482253) \times-1+(-0.462117157) \times 1-0.5=(-0.056968904)$ 
International Journal of Information Sciences and Techniques (IJIST) Vol.6, No.1/2, March 2016

The value produced by node 5 in $\mathrm{Eq}(9)$ will be squashed by the linear activation function i.e. the threshold function. Since, in the output layer the neuron present exhibits linear characteristics in minimum configuration MLFFN. Definition of Threshold function is :-

$¥(x)= \begin{cases}0, & x<\text { threshold } \\ 1, & x \geq \text { threshold }\end{cases}$

Here, threshold $=0$. From $\mathrm{Eq}(9)$ and $\mathrm{Eq}(10)$ the value of $\mathrm{y}$ when $\mathrm{x} 1=0$ and $\mathrm{x} 2=0$ will be $y=¥(-0.056968904)=0 \ldots \ldots$ (11) Since $(-0.056968904<0)$

$€(x)= \begin{cases}0, & x<\text { threshold } \\ 1, & x \geq \text { threshold }\end{cases}$

(12) $[$ threshold $=0$ for $\mathrm{Eq}(12)]$

From Eq. (11) the value of node 6 will be node $6=y \times-1+1 \times 0$

Therefore, here node $6=0 \times-1+1 \times 0=0$

From, (12) and (14) the value of output $\mathrm{z}$ for input $\mathrm{x} 1=0$ and $\mathrm{x} 2=0$ will be 1 , $z=€(0)=1$ (15) Since $(0=0)$

CASE2:- $[\mathrm{x} 1=1$ and $\mathrm{x} 2=0$ ]

At nodel the signal value will be from $\mathrm{Eq}(1)=1+0-1.5=(-0.5)$

At node2 the signal value will be from $\mathrm{Eq}(3)=1+0-0.5=0.5$

At node3 the signal value will be from $\mathrm{Eq}(16) \& \mathrm{Eq}(5)$ 
International Journal of Information Sciences and Techniques (IJIST) Vol.6, No.1/2, March 2016

node $3=\Psi(-0.5)=\frac{1-e^{-2 \times(-0.5)}}{1+e^{-2 \times(-0.5)}}=\frac{-1.718281828}{3.718281828}=(-0.462117157)$

At node4 the signal value will be from $\mathrm{Eq}(17) \& \mathrm{Eq}(5)$

node $4=\Psi(0.5)=\frac{1-e^{-2 \times(0.5)}}{1+e^{-2 \times(0.5)}}=\frac{0.632120558}{1.367879441}==(0.462117156)$

At node5 the signal value will be from $\mathrm{Eq}(18), \mathrm{Eq}(19) \& \mathrm{Eq}(8)=$

node $5=(-0.462117157) \times-1+(0.462117156) \times 1-0.5 \times 1=(0.424234313)$

Therefore the value of $\mathrm{y}$ for $\mathrm{x} 1=1$ and $\mathrm{x} 2=0$ from $\mathrm{Eq}(20)$ and $\mathrm{Eq}(10)$ is

$y=¥(0.424234313)=1 \ldots .(21)$ Since $0.424234313>0$

From $\mathrm{Eq}(14)$ the value of node 6 will be node $6=1 \times-1+0 \times 1=-1 \ldots \ldots$ (2)

From $\mathrm{Eq}(12)$ and $\mathrm{Eq}(22)$ the output $\mathrm{z}$ for the input $\mathrm{x} 1=1$ and $\mathrm{x} 2=0$ will be 0

$z=€(-1)=0$

(23) Since $(-1<0)$

CASE 3:- $[\mathrm{x} 1=0$ and $\mathrm{x} 2=1]$

At nodel the signal value will be from $\mathrm{Eq}(1)=0+1-1.5=(-0.5) \ldots \ldots$ (24)

At node2 the signal value will be from $\mathrm{Eq}(3)=0+1-0.5=(0.5) \ldots \ldots$ (25)

At node3 the signal value will be from $\mathrm{Eq}(24) \& \mathrm{Eq}(5)$

$\Psi(-0.5)=\frac{1-e^{-2 \times(-0.5)}}{1+e^{-2 \times(-0.5)}}=\frac{-1.718281828}{3.718281828}=(-0.462117157) \ldots \ldots$ 
International Journal of Information Sciences and Techniques (IJIST) Vol.6, No.1/2, March 2016

At node4 the signal value will be from $\mathrm{Eq}(25) \& \mathrm{Eq}(5)$

$\Psi(0.5)=\frac{1-e^{-2 \times(0.5)}}{1+e^{-2 \times(0.5)}}=\frac{0.632120558}{1.367879441}=(0.46217156)$

At node5 the signal value will be from $\mathrm{Eq}(26), \mathrm{Eq}(27) \& \mathrm{Eq}(8)=$

$(-0.462117157 \times-1)+(0.462117156 \times 1)-0.5 \times 1=(0.424234313)$

Therefore the value of $\mathrm{y}$ for $\mathrm{x} 1=0$ and $\mathrm{x} 2=1$ from $\mathrm{Eq}(22)$ and $\mathrm{Eq}(10)$ is

$y=¥(0.424234313)=1$ (29) Since $(0.424234313>0)$

From $\mathrm{Eq}(14)$ the value of node 6 will be node $6=1 \times-1+0 \times 1=-1$

From $\mathrm{Eq}(12)$ and $\mathrm{Eq}(30)$ the output $\mathrm{z}$ for the input $\mathrm{x} 1=0$ and $\mathrm{x} 2=1$ will be 0

$z=€(-1)=0$

(31) Since $(-1<0)$

CASE 4:- $[\mathrm{x} 1=1$ and $\mathrm{x} 2=1]$

At nodel the signal value will be from $\mathrm{Eq}(1)=(1+1-1.5)=(0.5)$

At node2 the signal value will be from $\mathrm{Eq}(3)=(1+1-0.5)=(1.5)$

At node 3 the signal value will be from $\mathrm{Eq}(32) \& \mathrm{Eq}(5)$

$\Psi(0.5)=\frac{1-e^{-2 \times 0.5}}{1+e^{-2 \times 0.5}}=\frac{0.632120558}{1.367879441}=(0.462117156)$

At node4 the signal value will be from $\mathrm{Eq(33)}$ and $\mathrm{Eq}(5)$

node $4=\Psi(1.5)=\frac{1-e^{-2 \times(1.5)}}{1+e^{-2 \times(1.5)}}=\frac{0.950212931}{1.049787068}=(0.905148253)$

At node5 the signal value will be from $\mathrm{Eq}(34), \mathrm{Eq}(35)$ and $\mathrm{Eq}(8)=$

$=(0.462117156 \times-1)+(0.905148253 \times 1)-0.5==(-0.056968902)$

Therefore, the value of $\mathrm{y}$ when $\mathrm{x} 1=1$ and $\mathrm{x} 2=1$ will be from $\mathrm{Eq}(36)$ and $\mathrm{Eq}(10)$

$y=¥(-0.056968902)=0$ (37) Since $(-0.056968902<0)$

From $\mathrm{Eq}(14)$ the value of node 6 will be node $6=0 \times-1+0 \times 1=0$

From $\mathrm{Eq}(12)$ and $\mathrm{Eq}(38)$ the output $\mathrm{z}$ for the input $\mathrm{x} 1=1$ and $\mathrm{x} 2=1$ will be 0

$z=€(0)=1$

(39) Since $(0=0)$

From Equations $\mathrm{Eq}(15), \mathrm{Eq}(23), \mathrm{Eq}(31)$ and $\mathrm{Eq}(39)$ the first solution provided is proved to be a valid solution. 


\subsection{Second Proposed Solution to the Ex-NOR problem}

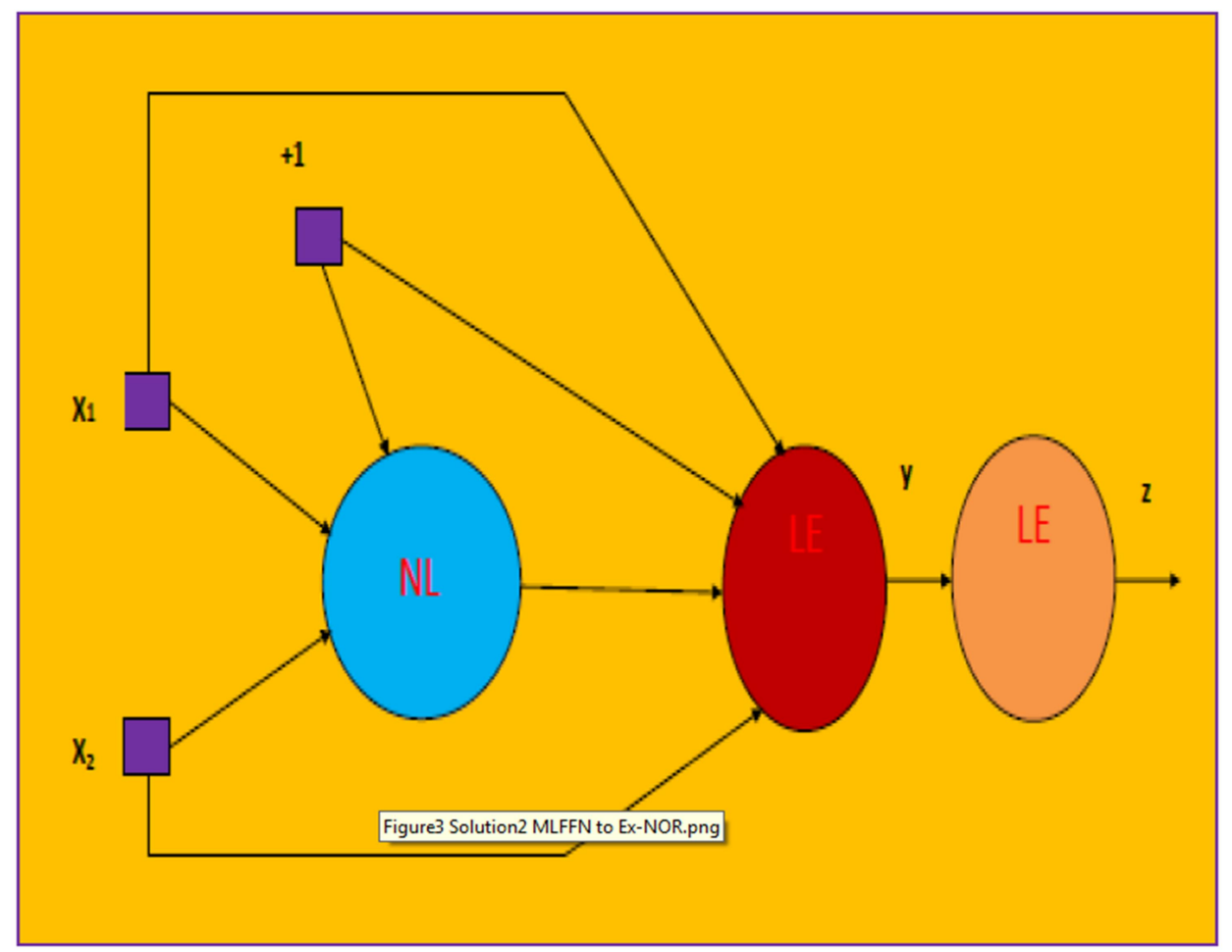

Figure 3. Architectural graph representing the second solution to the Ex-NOR problem using MLFFN

In this section we will see the second proposed solution to the Ex-NOR problem. The proposed solution is a MLFFN comprising of three layers. Each layer comprises of one neuron each. The first layer comprises of one neuron. The neuron in layer one is non-linear. The neuron in layer two and three are linear in nature. Figure 3 and Figure 4 represent Architectural Graph and Signal Flow Graph for the proposed solution. 


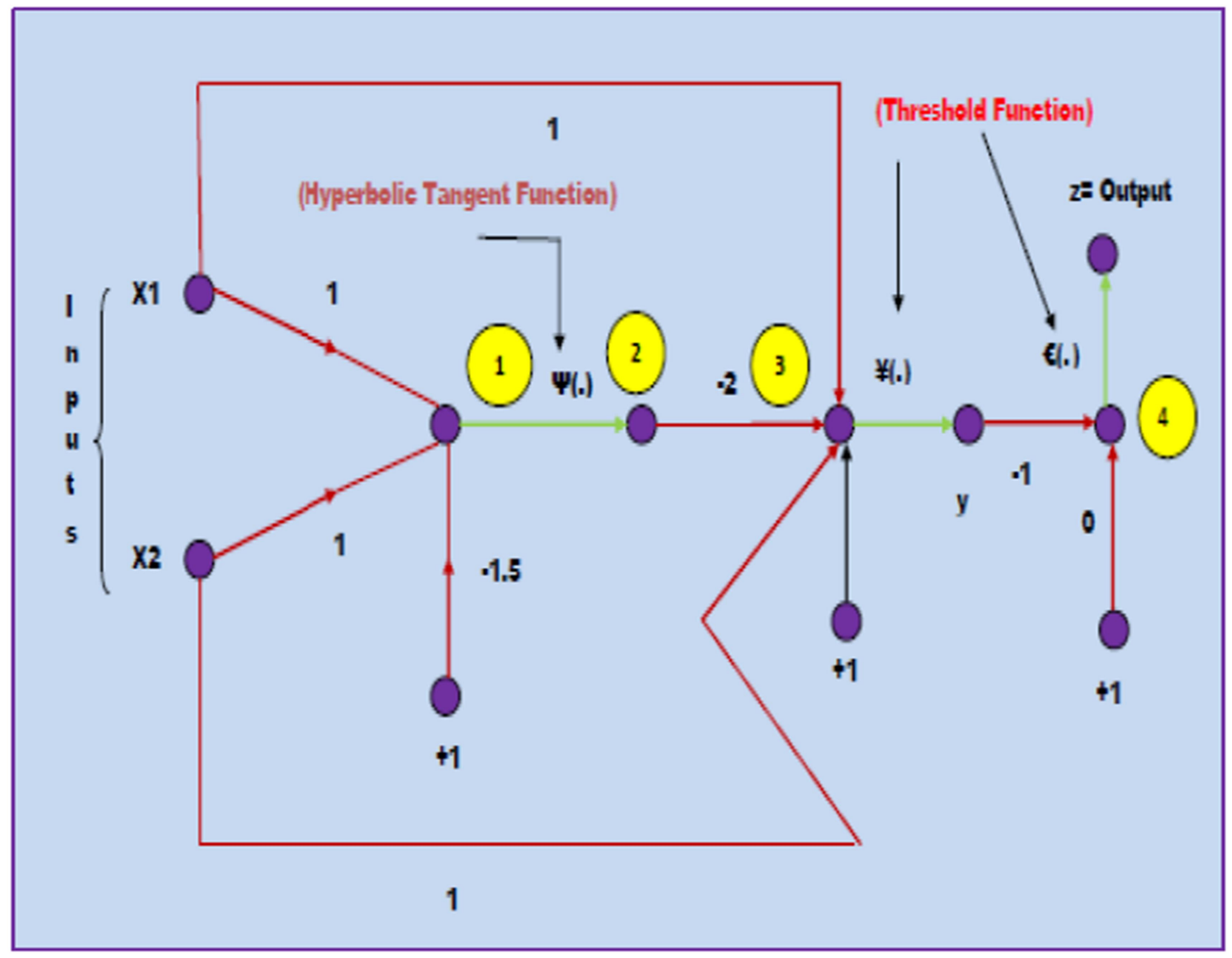

Figure4. Signal flow graph representing the second solution to the Ex-NOR problem using MLFFN 
International Journal of Information Sciences and Techniques (IJIST) Vol.6, No.1/2, March 2016

CASE 1:- $[\mathrm{x} 1=0$ and $\mathrm{x} 2=0$ ]

At node1 the value of the signal will be $=x 1 \times 1+x 2 \times 1+1 \times(-1.5)$

node $1=0 \times 1+0 \times 1+(-1.5)=(-1.5)$

The Activation function used in this case for hidden layer having non-linear element is hyperbolic tangent function. The definition of hyperbolic tangent function is:-

$\tan h x=\frac{\sin h x}{\cos h x}=\frac{e^{x}-e^{-x}}{e^{x}+e^{-x}}=\frac{e^{2 x}-1}{e^{2 x}+1}=\frac{1-e^{-2 x}}{1+e^{-2 x}} \ldots \ldots \ldots \ldots \ldots(c)$

Where, $\mathrm{e}=2.718281828456$ (constant) is the natural logarithm base also known as Euler's number, $\mathrm{x}$ is the induced local field of the neuron and Range $[-1 .+1]$.

At node2 the value of the signal will be from $\mathrm{Eq}(\mathrm{b}) \& \mathrm{Eq}(\mathrm{c})$

node $2=\Psi(-1.5)=\frac{1-e^{-2 \times(-1.5)}}{1+e^{-2 \times(-1.5)}}=\frac{-19.08553692}{21.08553692}=(-0.905148253) \ldots(d)$

At node 3 the value of the signal will be from $\operatorname{Eq}(d)$

node $3=x 1 \times 1+x 2 \times 1+$ node $2 \times-2+1 \times(-0.5) \ldots . .(e)$

node $3=0 \times 1+0 \times 1+(-0.905148253 \times-2)+1 \times(-0.5)$

node $3=(1.810296508-0.5)=(1.310296508) \ldots .(f)$

In this case for output neuron which is linear element we will be using threshold function as the activation function. The threshold value is 1.35 . The definition of Threshold function is:- 
International Journal of Information Sciences and Techniques (IJIST) Vol.6, No.1/2, March 2016

In this case for output neuron which is linear element we will be using threshold function as the activation function. The threshold value is 1.35 . The definition of Threshold function is:-1

$¥(x)=\left\{\begin{array}{l}1 \text { if } x \geq \text { threshold } \\ 0 \text { if } x<\text { threshold }\end{array}\right.$

Here, $\mathrm{x}=$ induced local field value.From $\mathrm{Eq}(\mathrm{f})$ and $\mathrm{Eq}(\mathrm{g})$ we get the value of $\mathrm{y}$ for $\mathrm{xl}=0$ and $\mathrm{x} 2=0$ as

$y=¥(1.310296508)=0$

(h) Since $(1.310296508<1.35)$

$€(x)= \begin{cases}0, & x<\text { threshold } \\ 1, & x \geq \text { threshold }\end{cases}$

(i) $[$ threshold $=0$ for $\mathrm{Eq}(i)]$

From Eq. $(h)$ the value of node4 will be node $4=y \times-1+1 \times 0 \ldots \ldots .(j)$

Therefore, here node $6=0 \times-1+1 \times 0=0$

From $(i)$ and $(k)$ the value of output $\mathrm{z}$ for input $\mathrm{x} 1=0$ and $\mathrm{x} 2=0$ will be 1 ,

$z=€(0)=1$ (l) Since $(0=0)$

\section{CASE 2:-}

$\mathrm{x} 1=1$ and $\mathrm{x} 2=0$

At node1 value of the signal will be from $\operatorname{Eq}(a)=1+0-1.5=(-0.5)$ 
International Journal of Information Sciences and Techniques (IJIST) Vol.6, No.1/2, March 2016

At node2 value of the signal will be from $\mathrm{Eq}(m) \& \mathrm{Eq}(c)$

node $2=\Psi(-0.5)=\frac{1-e^{-2 \times(-0.5)}}{1+e^{-2 \times(-0.5)}}=\frac{-1.718281828}{3.718281828}=(-0.462117157)$

At node3 value of the signal will be from $\operatorname{Eq}(n) \& \mathrm{Eq}(e)$

node $3=1+0+(-2 \times-0.462117157)-0.5=(1.424234314)$

The value of $y$ for $\mathrm{x} 1=1$ and $\mathrm{x} 2=0$ will be from $\mathrm{Eq}(o) \& \mathrm{Eq}(g)$

$y=¥(1.424234314)=1 \ldots \ldots \ldots(p)$ Since $(1.424234314>1.35$

From $\mathrm{Eq}(j)$ the value of node 4 will be node $4=1 \times-1+0 \times 1=-1 \ldots \ldots(q)$

From $\mathrm{Eq}(i)$ and $\mathrm{Eq}(q)$ the output $z$ for the input $\mathrm{x} 1=1$ and $\mathrm{x} 2=0$ will be 0

$z=€(-1)=0$

(r) Since $(-1<0)$

CASE 3:- $[\mathrm{x} 1=0$ and $\mathrm{x} 2=1]$

At node1 the value of signal from $\operatorname{Eq}(a)=(0+1-1.5)=(-0.5)$

At node2 the value of signal from $\mathrm{Eq}(s) \& \mathrm{Eq}(c)$

node $2=\Psi(-0.5)=\frac{1-e^{-2 \times(-0.5)}}{1+e^{-2 \times(-0.5)}}=\frac{-1.718281828}{3.718281828}=(-0.462117157)$

At node 3 the value of the signal from $\mathrm{Eq}(t)$ and $\mathrm{Eq}(e)$

node $3=(0+1+(-2 \times-0.462117157)-0.5=(1.424234314)$

Therefore the value of $y$ for the input $\mathrm{x} 1=0$ and $\mathrm{x} 2=1$ will be from $\mathrm{Eq}(u)$ and $\mathrm{Eq}(g)$

$y=¥(1.424234314)=1$

(v) Since $(1.424234314>1.35)$

From $\mathrm{Eq}(j)$ the value of node 6 will be node $4=1 \times-1+0 \times 1=-1 \ldots \ldots(w)$

From $\mathrm{Eq}(i)$ and $\mathrm{Eq}(w)$ the output $z$ for the input $\mathrm{x} 1=0$ and $\mathrm{x} 2=1$ will be 0

From Equations $\mathrm{Eq}(l), \mathrm{Eq}(r), \mathrm{Eq}(x)$ and $\mathrm{Eq}(D)$ the first solution provided is proved to be a valid solution. 


\subsection{Third Proposed Solution to the Ex-NOR problem}

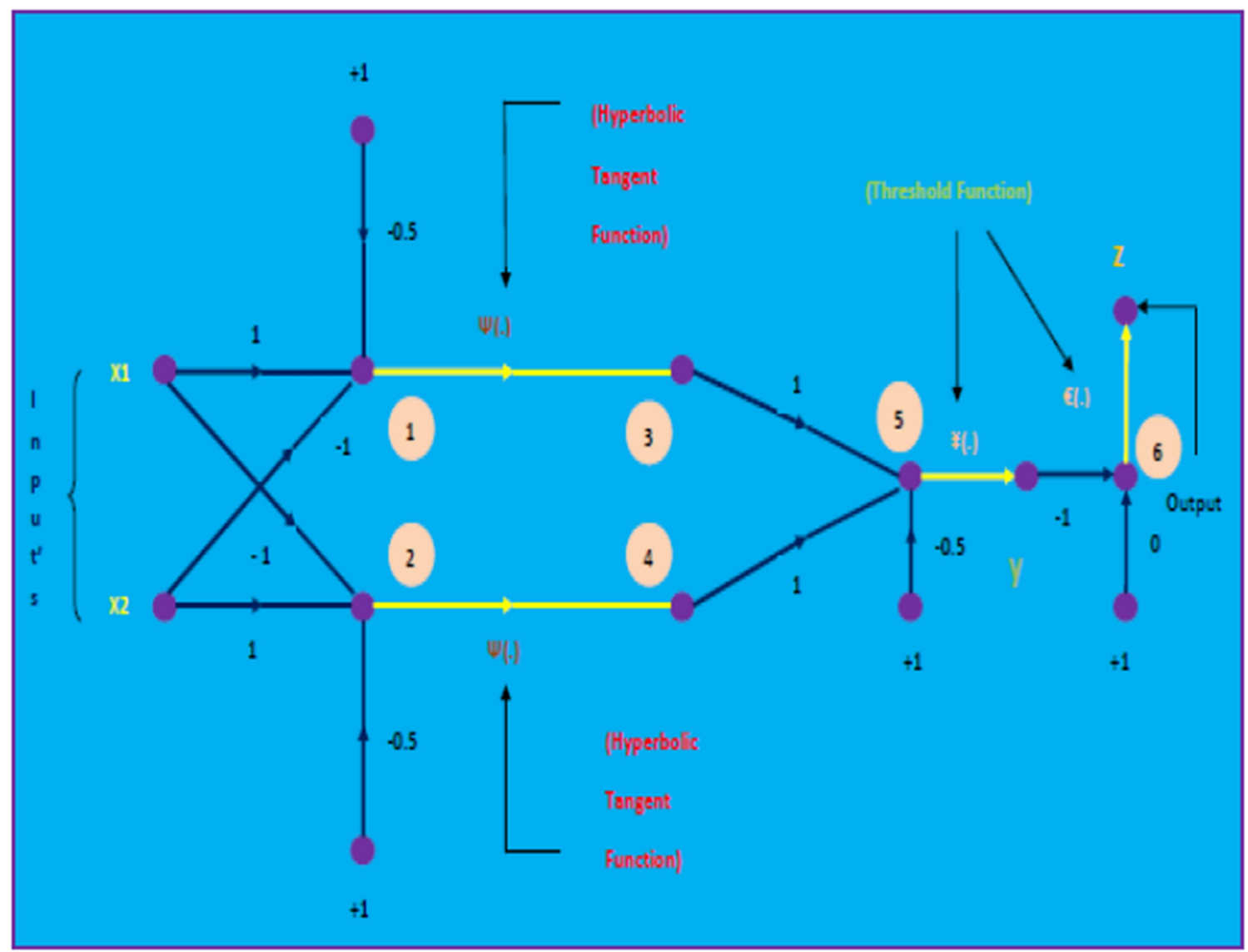

Figure5. Signal flow graph representing the third solution to the Ex-NOR problem using MLFFN

In Third proposed solution a MLFFN is proposed which comprises of three layers. The First layer comprises of two neurons. The second layer comprises of one neuron. The third layer comprises of one neuron. The first layer comprises of non-linear neuron. The second and third layer comprises of neurons which are having linear in nature. The neurons are characterized linear and non-linear on the basis of the characteristic they exhibit. The naming of the network could be (22-1-1). The solution proposed specifies the final values for weights acquired after training the network. After attaining the values prescribed in the solution the proposed ANN gives the desired values for output for the applicable input to the Ex-NOR logic GATE. In this section we will see the mathematical proof of the provided solution. Figure 1 represents the proposed Architectural Graph for the problem, whereas the Figure 5 represents the Signal Flow Graph giving the detail of the weights and other parameters involved in the ANN. In the Architectural graph NL represents nonlinear whereas LE represents Linear element. $\mathrm{Z}$ represents the output produced by the ANN. 
International Journal of Information Sciences and Techniques (IJIST) Vol.6, No.1/2, March 2016

CASE 1:- When $\mathrm{x} 1=0$ and $\mathrm{x} 2=0$,

At node 1 value of signal will be $x 1 \times 1+x 2 \times-1+1 \times(-0.5)$

$=0 \times 1+0 \times-1+1 \times(-0.5)=(-0.5)$.

At node 2 Signal value will be $x 1 \times-1+x 2 \times 1+1 \times(-0.5)$

$=0 \times-1+0 \times 1+1 \times(-0.5)=(-0.5)$

Here, the activation function used for hidden layer is Hyperbolic tangent function. The Def. of which is given below:-

$\tan h x=\frac{\sin h x}{\cos h x}=\frac{\left(e^{x}-e^{-x}\right)}{\left(e^{x}+e^{-x}\right)}=\frac{\left(\mathrm{e}^{2 \mathrm{x}}-1\right)}{\left(\mathrm{e}^{2 \mathrm{x}}+1\right)}=\frac{\left(1-e^{-2 x}\right)}{\left(1+e^{-2 x}\right)}$ here $e=2.718281828456 \ldots$

$\mathrm{x}=$ induced local field value, $\mathrm{e}=$ The natural logarithm base also known as Euler's number \& Range $=[-1,+1]$

At node 3 the signal value will be from $\mathrm{Eq}(2$ and 5)

$=\Psi(-0.5)=\frac{\left(1-e^{-2 \times-0.5}\right)}{\left(1+e^{-2 \times-0.5}\right)}=\frac{-1.718281828}{3.718281828}=(-0.462117157)$

At node 4 the signal value will be from Eq (4 and 5)

$=\Psi(-0.5)=\frac{\left(1-e^{-2 \times-0.5}\right)}{\left(1+e^{-2 \times-0.5}\right)}=\frac{-1.718281828}{3.718281828}=(-0.462117157)$

At node 5 the signal value will be from Eq 6 and $\mathrm{Eq} 7$

$=$ node $3 \times 1+$ node $4 \times 1+1 \times(-0.5) \ldots \ldots \ldots \ldots \ldots(8 a)$

$=(-0.462117157) \times 1+(-0.462117157) \times 1+1 \times(-0.5)=(-1.424234314) \ldots(9 a)$

In the output layer the function used is Threshold function. The Definition of the threshold function is given below:-

$¥(x)=\left\{\begin{array}{l}1 \text { if } x \geq \text { threshold } \\ 0 \text { if } x<\text { threshold }\end{array}\right.$ here threshold value is set to value -1

$y=¥(-1.424234314)=0$ Since,$((-1.424234314)<-1)$

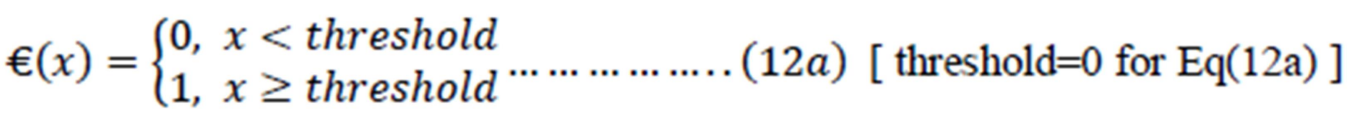


International Journal of Information Sciences and Techniques (IJIST) Vol.6, No.1/2, March 2016

CASE 2:- When $\mathrm{x} 1=1$ and $\mathrm{x} 2=0$,

At node 1 value of signal will be $x 1 \times 1+x 2 \times-1+1 \times(-0.5)$

Substituting the value of $x 1$ and $x 2=1 \times 1+0 \times-1+1 \times(-0.5)=(0.5)$

At node 2 Signal value will be $x 1 \times-1+x 2 \times 1+1 \times(-0.5)$,

Substituting the value of $x 1$ and $x 2=1 \times-1+0 \times 1+1 \times(-0.5)=(-1.5) \ldots(17 a)$

At node 3 the signal value will be from $\mathrm{Eq}(16 \mathrm{a}$ and $5 \mathrm{a})$

$=\Psi(0.5)=\frac{\left(1-e^{-2 \times 0.5}\right)}{\left(1+e^{-2 \times 0.5}\right)}=\frac{0.632120558}{1.367879441}=(0.462117156)$

At node 4 the signal value will be from Eq (17a and 5a)

$$
=\Psi(-1.5)=\frac{\left(1-e^{-2 \times-1.5}\right)}{\left(1+e^{-2 \times-1.5}\right)}=\frac{-19.08553692}{21.08553693}=(-0.905148253)
$$

At node 5 the signal value will be from Eq 18a, Eq. 19a and Eq 8a

$=(0.462117156) \times 1+(-0.905148253) \times 1+1 \times(-0.5)=(-0.943031097) \ldots(20 a)$

From $\mathrm{Eq}(20 \mathrm{a})$ and $\mathrm{Eq}(10 \mathrm{a})$ the value of $\mathrm{y}$ for the second case i.e. $\mathrm{x} 1=1$ and $\mathrm{x} 2=0$ will be $¥(-0.943031097)=1$ Since,$((-0.943031097)>-1)$

From $\mathrm{Eq}(14 \mathrm{a})$ the value of node 6 will be node $6=1 \times-1+0 \times 1=-1 \ldots \ldots$ (22a)

From $\mathrm{Eq}(12 \mathrm{a})$ and $\mathrm{Eq}(22 \mathrm{a})$ the output $\mathrm{z}$ for the input $\mathrm{x} 1=1$ and $\mathrm{x} 2=0$ will be 0 
International Journal of Information Sciences and Techniques (IJIST) Vol.6, No.1/2, March 2016

$z=€(-1)=0$ (23a) Since $(-1<0)$

CASE 3:- When $\mathrm{x} 1=0$ and $\mathrm{x} 2=1$,

At node 1 value of signal will be $x 1 \times 1+x 2 \times-1+1 \times(-0.5)$

Substituting the value of $x 1$ and $x 2=0 \times 1+1 \times-1+1 \times(-0.5)=(-1.5) \ldots \ldots(24 a)$

At node 2 Signal value will be $x 1 \times-1+x 2 \times 1+1 \times(-0.5)$

Substituting the value of $x 1$ and $x 2=0 \times-1+1 \times 1+1 \times(-0.5)=(0.5) \ldots \ldots(25 a)$

At node 3 the signal value will be from $\mathrm{Eq}(24 \mathrm{a}$ and $5 \mathrm{a})$

$=\Psi(-1.5)=\frac{\left(1-e^{-2 \times-1.5}\right)}{\left(1+e^{-2 \times-1.5}\right)}=\frac{-19.08553692}{21.08553693}=(-0.905148253)$

At node 4 the signal value will be from $\mathrm{Eq}(25 \mathrm{a}$ and $5 \mathrm{a})$

$=\Psi(0.5)=\frac{\left(1-e^{-2 \times 0.5}\right)}{\left(1+e^{-2 \times 0.5}\right)}=\frac{0.632120558}{1.367879441}=(0.462117156)$

At node 5 the signal value will be from Eq 26a, Eq 27a and Eq 8a

$=(-0.905148253) \times 1+(0.462117156) \times 1+1 \times(-0.5)=(-0.943031097)$

From $\mathrm{Eq}(28 \mathrm{a})$ and $\mathrm{Eq}(10 \mathrm{a})$ the value of $\mathrm{y}$ for the third case i.e. $\mathrm{x} 1=0$ and $\mathrm{x} 2=1$ will be $y=¥(-0.943031097)=1$ Since,$((-0.943031097)>-1)$ 
From $\mathrm{Eq}(14 \mathrm{a})$ the value of node6 will be node $6=1 \times-1+0 \times 1=-1 \ldots \ldots(30 a)$

From $\mathrm{Eq}(12 \mathrm{a})$ and $\mathrm{Eq}(30 \mathrm{a})$ the output $\mathrm{z}$ for the input $\mathrm{x} 1=0$ and $\mathrm{x} 2=1$ will be 0

$$
z=€(-1)=0 \ldots \ldots \ldots \ldots \ldots \ldots(31 a) \text { Since }(-1<0)
$$

CASE 4:- When $\mathrm{x} 1=0$ and $\mathrm{x} 2=1$,

At node 1 value of signal will be $=x 1 \times 1+x 2 \times-1+1 \times(-0.5)$

Substituting the value of $x 1$ and $x 2=1 \times 1+1 \times-1+1 \times(-0.5)=(-0.5) \ldots(32 \mathrm{a})$

At node 2 Signal value will be $x 1 \times-1+x 2 \times 1+1 \times(-0.5)$,

Substituting the value of $x 1$ and $x 2=1 \times-1+1 \times 1+1 \times(-0.5)=(-0.5) \ldots . .(33 a)$

At node 3 the signal value will be from $\mathrm{Eq}(32 \mathrm{a}$ and $5 \mathrm{a})$

$=\Psi(-0.5)=\frac{\left(1-e^{-2 \times-0.5}\right)}{\left(1+e^{-2 \times-0.5}\right)}=\frac{-1.718281828}{3.718281828}=(-0.462117157)$

At node 4 the signal value will be from $\mathrm{Eq}(33 \mathrm{a}$ and $5 \mathrm{a}$ )

$=\Psi(-0.5)=\frac{\left(1-e^{-2 \times-0.5}\right)}{\left(1+e^{-2 \times-0.5}\right)}=\frac{-1.718281828}{3.718281828}=(-0.462117157)$

At node 5 the signal value will be from Eq 34a, Eq 35a and Eq 8a

$=(-0.462117157) \times 1+(-0.462117157) \times 1+1 \times(-0.5)=(-1.424234314) \ldots$

From $\mathrm{Eq} 36 \mathrm{a}$ and $\mathrm{Eq} 10 \mathrm{a}$ the value of the output $\mathrm{y}$ for input $\mathrm{x} 1=1$ and $\mathrm{x} 2=1$ will be

$$
y=¥(-1.424234314)=0 \text { Since, }((-1.424234314)<-1) . .(37 a)
$$

From $\mathrm{Eq}(14 \mathrm{a})$ the value of node 6 will be node $6=0 \times-1+0 \times 1=0 \ldots \ldots(38 a)$

From $\mathrm{Eq}(12 \mathrm{a})$ and $\mathrm{Eq}(38 \mathrm{a})$ the output $\mathrm{z}$ for the input $\mathrm{x} 1=1$ and $\mathrm{x} 2=1$ will be 0

$$
z=€(0)=1 \ldots \ldots \ldots \ldots \ldots \ldots(39 a) \text { Since }(0=0)
$$

From Equations $\mathrm{Eq}(15 \mathrm{a}), \mathrm{Eq}(23 \mathrm{a}), \mathrm{Eq}(31 \mathrm{a})$ and $\mathrm{Eq}(39 \mathrm{a})$ the third solution provided is proved to be a valid solution

\section{Conclusions}

In this paper two solutions to the Ex-NOR problem is proposed using the MLFFN. From the proposed solutions it is clear that it is possible to solve non-linearly separable problem using Multilayer Network. All the proposed solutions comprises of three layers where the elements in the network may be of either type i.e. Non-linear or Linear. The mathematical proof satisfies the condition for Ex-NOR. 
International Journal of Information Sciences and Techniques (IJIST) Vol.6, No.1/2, March 2016

\section{ACKNOWLEDGEMENTS}

I would like to place my thanks to my mother Smt Sushma Singh, my wife Smt Shubhra Singh, my brothers Abhinav Kant Singh and Abhuday Kant Singh, my sister Priyamvada Singh , my daughter Akshi and son Aishwaryat for the support that they give for the preparation of this work. Last but not the least I want to thank my Father Late Shri Triveni Kant Singh for his blessing, which enabled me to this work.

\section{REFERENCES}

[1] Vaibhav Kant Singh, (2015) "One Solution to XOR problem using Multilayer Perceptron having Minimum Configuration" International Journal of Science and Engineering, Vol. 3, No. 2, pp 32-41.

[2] Vaibhav Kant Singh, (2015) "Two Solutions to the XOR Problem using minimum configuration MLP”, International Journal of Advanced Engineering Science and Technological Research, Vol. 3, No. 3, pp16-20.

[3] Vaibhav Kant Singh (2016) "ANN Implementation of Constructing Logic Gates Focusing On ExNOR", Innovations in Computation, Communication and Cybernetics, Proceeding of BITCON 2016, Department of Computer Science \& Engineering, BIT, Durg, Chhattisgarh, India.

[4] Vaibhav Kant Singh (2016) "Mathematical Analysis For Training ANNs Using Basic Learning Algorithms", Innovations in Computation, Communication and Cybernetics, Proceeding of BITCON 2016, Department of Computer Science \& Engineering, BIT, Durg, Chhattisgarh, India.

[5] Vaibhav Kant Singh, (2016) "Proposing solution to XOR problem using minimum configuration MLP", To Appear Procedia of Elsevier, International Conference on Computational Modeling and Security (CMS 2016), Bangalore, India.

[6] Vaibhav Kant Singh and Shweta Pandey, (2016) "Minimum Configuration MLP for Solving XOR Problem," To Appear in IEEE Explore, Proceeding of the 10th INDIACom: INDIACom-2016, 3rd 2016 International Conference on "Computing for Sustainable Global Development," BVICAM, New Delhi, India.

\section{Author}

Vaibhav Kant Singh, [LM ISTE] and [LM CSI] is currently working as Assistant Professor in the Department of Computer Science \& Engineering, Institute of Technology, Guru Ghasidas Vishwavidyalaya, (Central University), Bilaspur, (C.G.), India. He is having around Eight years of teaching experience. $\mathrm{He}$ is having papers published in both international and national conferences. His papers are published in national and international journals too. His Papers are published by Publishers like Springer, ACM, AIP, WASET, Recently one

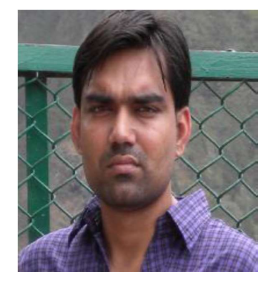
paper Accepted for Publication in Elsevier, One paper accepted in first round for publication in IEEE Explore etc. In International Conferences papers are published in Conferences organized by IIT's, IIIT's, CSI, WASET etc. He has taught several subjects like, AI and Expert System, Data Mining, Soft Computing (ANN, Genetic Algorithm and Fuzzy Logic), Network Programming, Compiler Design, Advanced Programming through JAVA, OOP through C++, Prolog, Lisp, Internet Fundamentals and Application, Parallel Computing, Operating System,DAA etc. 